\title{
REVIEW
}

\section{Pro/Con debate: Are barrier precautions cost-effective in improving patient outcomes in the intensive care unit?}

\author{
Nisha Thampi' and Andrew M Morris*
}

\begin{abstract}
You are responsible for a large medical surgical ICU. Your hospital administration has been very focused on reducing rates of hospital-acquired infections particularly in the wake of increasing public attention. However, it is time for budget preparation and your financial officer is concerned about the escalating costs associated with patient isolation and barrier precautions/personal protective equipment. Having become aware of the high costs associated with these interventions, you start to wonder about the wisdom of spending so much in this area. Your hospital administration wants your direction on next year's expenditures. You are debating whether the expense is worthwhile and advise your hospital administration accordingly.
\end{abstract}

\section{Statement for debate}

Infection prevention and control interventions are costeffective and improve patient outcomes in critically ill patients in the ICU.

\section{Introduction}

In the last 50 years, the discipline of infection prevention and control (IPAC) has undergone numerous metamorphoses. Initially intended to address nosocomial infections at the time of a nationwide epidemic of hospital-based staphylococcal infections [1], the practice of IPAC has shifted from that of reaction to prevention. There has been increasing emphasis on prevention of nosocomial infections through principles of hand hygiene, cleaning and sterilization, and antimicrobial

*Correspondence: amorris@mtsinai.on.ca

${ }^{2}$ Division of Infectious Diseases, Department of Medicine, Mount Sinai Hospital and University Health Network; Department of Medicine, University of Toronto; Mount Sinai Hopsital, 600 University Avenue, Suit 415, Toronto, ON M5G 1X5, Canada Full list of author information is available at the end of the article prophylaxis. Nonetheless, infectious diseases continue to challenge the quality of care provided in ICUs. Prompted by accreditation and patient-centered care, hospitals are challenged to provide high-quality care with finite financial resources. Any new IPAC initiative will thus be examined for its cost-effectiveness.

The broad impact of infection control on rates of hospital-associated infections goes back more than 200 years. A 1795 treatise on puerperal fever by Alexander Gordon, who made seminal observations as an obstetrician in the United Kingdom, suggested the illness was due to an infection, and recommended that healthcare workers $(\mathrm{HCW})$ wash themselves and have their clothing fumigated after contact with these patients [2]. The eminent American physician Oliver Wendell Holmes also suggested that gowns worn by physicians during autopsies served as vectors for transmission of puerperal fever among women in labor in the 1800s [3].

Ignaz Semmelweiss, a Hungarian physician and contemporary of Holmes, consolidated these theories through his epidemiologic work at a Viennese hospital with the realization that frequent contact with cadavers among medical students was associated with transmission of particles to women in labor, leading to puerperal fever and even sepsis. He is credited with introducing hand disinfection prior to vaginal examination, with a subsequent dramatic decrease in mortality [4]. This practice was reinforced in Florence Nightingale's 1859 treatise, in which she called for 'every nurse ... to be careful to wash her hands very frequently during the day' [5].

While the principles of hand hygiene still apply from the time of Semmelweiss, IPAC's scope has broadened from controlling infection with highly pathogenic organisms to preventing the spread of emerging drugresistant organisms and viruses. The escalating costs of modern patient isolation and personal protective equipment, however, especially among critically ill patients in the ICU, call into question the wisdom of spending so much in this area. Most importantly, are these expenses justified in terms of positive patient 
outcomes? To address this question, we present a discussion on the merits, concerns and costs surrounding enhanced precautions for critically ill patients, the latter of which was found to be lacking in a systematic review of the literature performed within the last 5 years [6].

\section{Pro: IPAC interventions are cost-effective and improve patient outcomes in critically ill patients in the ICU}

Nosocomial infections in the ICU have been associated with significant mortality and increased resource consumption [7]. A recent study conducted prospectively across Austrian ICUs found that patients with nosocomial infections had prolonged device exposure, including endotracheal tubes, central venous catheters and urinary catheters, increased mortality rates, and longer length of stay in the ICU and the hospital [8].

To appreciate the impact of nosocomial infections in the ICU, one needs to look no further than the issue of methicillin-resistant Staphylococcus aureus (MRSA). During the 1990s, healthcare-associated MRSA infections increased dramatically [9] and MRSA was the predominant pathogen in most ICUs in the United States [10]. Moreover, these infections were associated with increased mortality and costs as compared with antibioticsusceptible strains of the same species [11,12]. Transmission has been the major factor contributing to the increased prevalence of MRSA, with a 15-fold higher rate of transmission of MRSA among patients on standard precautions who were not yet recognized to be colonized $[13,14]$.

The implementation of strict IPAC practices, including hand hygiene and active surveillance of colonized patients with subsequent barrier precautions, has been credited for the declining rates of MRSA catheter-related bloodstream infections by $50 \%$ or more in medical, surgical, cardiac and pediatric ICUs since 2001 [15]. The benefits of specific IPAC interventions, however, are difficult to tease out as many are bundled, especially those related to personal protective equipment and active surveillance. Hand hygiene has become a well-recognized means of lowering the rates of hospital-associated infections in ICU and non-ICU settings, despite highest compliance rates of only 55 to $65 \%$ [16-18]. Furthermore, two prospective studies in medical ICUs found that the use of gowns with gloves was associated with a lower risk of nosocomial cross-transmission of vancomycin-resistant Enterococcus (VRE) $[19,20]$. A large multicenter trial in 108 ICUs in Michigan saw a significant and durable decrease in the mean rate of catheter-related bloodstream infections with maximum sterile barrier precautions during insertion of central venous catheters and removal of unnecessary catheters [21]. Jain and colleagues also reported a significant decline in rates of healthcare-associated
MRSA infections in the largest prospective observational trial to date, with the implementation of an MRSA bundle in ICU and non-ICU settings across the United States [22].

In an effort to determine which intervention has the most significant impact on nosocomial infections, a retrospective study reviewed four major IPAC interventions in ICU and non-ICU settings for controlling MRSA: maximally sterile central venous catheter placement, alcohol hand rub, a hospital-wide hand hygiene campaign, and routine surveillance for MRSA in all ICU patients with implementation of barrier precautions if the patient was found to be positive [23]. Only a routine screen with subsequent contact isolation was associated with a significant decrease in hospital-associated MRSA bacteremia in the ICU setting. Active surveillance for MRSA colonization among ICU patients with subsequent implementation of barrier precautions has also been shown in the non-ICU setting [23-25] and in the neonatal ICU setting [13] to significantly decrease the rates of transmission and incidence of nosocomial MRSA bacteremias, despite only 55\% compliance with contact precautions for ICU patients known to be colonized with MRSA [25].

Against the extra costs and extra length of stay attributed to nosocomial infections among critically ill patients, IPAC interventions have been shown to be costeffective for bloodstream infections [7,26]. Kirkland and Weinstein estimated that the cost for a patient in contact isolation within the ICU, with highly-compliant disposable gown and latex glove use, was US\$1,627 per patient (1999 valuation) [27]. Broadening handwashing compliance to patients and relatives has been calculated to save more than CDN\$600,000 per year in a community hospital setting [28].

Numerous studies have shown that active surveillance (heterogeneously defined in studies as universal versus targeted screening of high-risk patients) with subsequent hand hygiene and use of gowns, gloves and/or masks is associated not only with decreased rates of nosocomial infections - especially due to MRSA and VRE - but with significant cost savings. Following two MRSA outbreaks in separate neonatal ICUs, Karchmer and colleagues evaluated the costs of one institution's preventive measures - namely, weekly active surveillance cultures and isolation of MRSA-colonized patients - with the potential costs of not intervening promptly or effectively at the other institution [29]. The overall cost of surveillance cultures was US\$27,590 (2002 valuation). The cost of isolation, which included gloves, paper gown, simple isolation mask and the 1 minute of personnel time to put on and remove the items, was estimated at US $\$ 0.94$ per patient per visit, for a total of US\$21,027 to 42,047 for the duration of the outbreak. The attributable 
excess costs of MRSA bacteremia infections in the other neonatal ICU was estimated at 19 -fold to 27 -fold higher, suggesting that the prevention of 2.8 to 4.0 MRSA bacteremias would have justified the costs of the preventive measures. A cost-benefit analysis in a medical ICU also showed that active identification of patients with infection or colonization by MRSA via selective screening and isolation was more cost-effective than treating patients with MRSA infections, with mean excess total costs attributable to nosocomial MRSA infection at $\$ 9,275$ per patient compared with a total cost of contact screening and isolation of up to $\$ 700$ per patient [30].

Similarly, the cost-effectiveness of active surveillance for all high-risk patients in one hospital was compared with estimated attributable costs due to VRE bacteremias in another hospital with 75 cases of VRE infections over a 24-month period [31]. The total cost of active surveillance cultures was US\$91,108, and the cost of contact isolation - including gown, gloves and labor - was US\$1.18 per patient per visit. Despite the excess attributable cost of VRE bacteremia being estimated from a case-control study of VRE, the cost of culture and isolation was found to be one-third the cost attributable to VRE bacteremia in an endemic setting. A similar study in a VRE-endemic oncology unit found that enhanced precautions, which included surveillance cultures, handwashing, and contact isolation with gown and glove use, resulted in fewer VRE bloodstream infections and colonized patients, with a net saving of US $\$ 189,318$ over 1 year [32]. The authors concluded that controlling VRE transmission was cost-effective, particularly in a setting where the incidence of VRE bloodstream infections is at least six to nine patients per year.

Despite the small literature base, existing evidence supports IPAC interventions as cost-effective in decreasing transmission of MRSA and VRE among critically ill patients.

\section{Con: Infection prevention and control interventions are not cost-effective and do not improve patient outcomes in critically ill patients in the ICU}

Despite published guidelines on hospital infection control practices in ICU and non-ICU settings, there remains skepticism on whether these measures are cost-effective or even detrimental to the quality of patient care.

A case-control study of patients in a medical ICU showed that HCW were highly compliant with gown and glove use, but were two times less likely to enter the rooms of patients in contact isolation and had significantly less direct contact with these patients [27]. In a prospective cohort study among 139 medical inpatients infected or colonized with VRE at two medical centers, attending physicians were about one-half as likely to examine patients under contact precautions compared with those not under precautions, and only examined one-third of patients in the isolation group during morning rounds [33]. A similar observational study among surgical ICU and non-ICU patients found a reduction in the time $\mathrm{HCW}$ spent with patients on contact precautions (5.3 versus 10.9 visits per hour for patients not isolated) not explained by severity of illness [34].

During the severe acute respiratory syndrome outbreak in Toronto, Canada, patients with the infection were frequently alone between brief contacts with staff, and some had worsening psychological symptoms during their stay, including panic attacks. Nearly one-half of hospital employees reported difficulty in communicating with patients through the enhanced infectious precautions $[35,36]$.

The emerging question of patient safety was raised in a study of patients isolated for MRSA colonization or infection [37]. In addition to dramatically reduced clinical documentation, these patients were twice as likely to experience preventable adverse events, including supportive care failures such as falls, pressure ulcers, and fluid or electrolyte disorders, and reported higher rates of dissatisfaction with the quality of care received. A prospective, randomized and controlled study in two surgical ICUs sought to determine whether barrier precautions would lower the incidence of hospitalassociated pneumonia in 153 intubated patients. Among intubated patients in two surgical ICUs, patients randomized to contact isolation with disposable gowns, gloves and hand hygiene had similar rates of airway colonization but a higher incidence of nosocomial pneumonia than patients on standard precautions with hand hygiene and gloves over a 15-month period [38].

Taken together, these studies suggest that isolation of patients for infection control purposes may have a deleterious effect on the quality of medical care received and perceived by these patients.

Another argument against enhanced infection control measures is that they are often based on poor-quality evidence. Given the high prevalence of influenzaassociated respiratory illnesses in hospitals, recent studies have examined the practice of enhanced respiratory precautions. A randomized controlled trial comparing surgical masks with N95 masks among HCW during the influenza season showed similar rates of laboratory-confirmed influenza [39]. A contemporary study among patients from an emergency department also found surgical masks to be equally as effective as N95 masks, with no influenza detected by PCR with either mask [40]. Moreover, many HCW find N95 masks difficult to tolerate [36]. Given that a recent literature review was unable to identify high-quality studies to 
support the use of N95 over surgical masks in healthcare settings [41], the lack of definitive data would suggest that the use of the more expensive N95 masks may not be justified in most healthcare settings.

Most of the evidence for controlling VRE transmission relates to colonization rather than bacteremia, and may not necessarily reflect improved patient outcomes. High colonization pressure (that is, the proportion of other patients colonized with VRE within a medical ICU) is a major variable affecting the rate of acquisition of VRE $[20,42]$. However, there have been no studies linking colonization pressure to rates of VRE-associated disease. While gown and glove use for preventing nosocomial transmission of VRE is a Grade IA recommendation by the Society for Healthcare Epidemiology of America [14], there is no clear evidence suggesting that these infection control practices have led to a reduction in the incidence of disease. Among ICU patients who acquired and did not acquire VRE, rates of hand-washing and glove use were comparable [42], and gowns may [20] or may not [43] have a protective effect over glove use alone. Furthermore, unlike MRSA, preventing VRE transmission has been suggested to be cost-effective only in endemic settings, where at least six patients per year will develop bloodstream infections [32]. Even then, compliance with barrier precautions may itself present as a barrier to reducing the rate of transmission of nosocomial infections. In a recent cluster-randomized controlled trial involving 5,434 admissions to 10 intervention ICUs and 3,705 admissions to eight control ICUs, there was no reported difference in rates of transmission of MRSA or VRE through the use of barrier precautions, in which surveillance for MRSA and VRE detection was performed in both groups and contact precautions were initiated only in the intervention group [44]. Barrier precautions were not followed as often as required by protocol, and it was suggested that this lack of adherence led to the lack of impact on transmission rates. Interestingly, this finding has not been demonstrated in other trials but is arguably reflective of real-world compliance issues around infection control strategies.

\section{Resolution: In the ICU, patient isolation and barrier} protection are more beneficial than detrimental

Infection control practices have made a remarkable impact on rates of cross-transmission among critically ill patients. Compliance with hand hygiene and contact precautions has been shown to be significantly higher in isolation rooms, suggesting that knowledge of enhanced precautions may motivate $\mathrm{HCW}$ to further decrease transmission to other patients $[19,20,27,43,45]$. Isolation with barrier precautions has decreased the spread of MRSA infection when coupled with active surveillance cultures to detect colonized patients serving as a reservoir for spread [24]. The costs of implementing enhanced precautions have also been shown to be effective in decreasing MRSA infection, although less clearly so regarding VRE bacteremia (but certainly for VRE colonization), and arguably excessive for influenza (given the data for surgical masks being non-inferior to the more expensive N95 masks).

Such IPAC interventions have been promoted as not only beneficial to the isolated patient but also to other patients and HCW in the ICU. However, the net effect of IPAC measures on patient care - with less time spent in patient rooms, less time examining patients, more incomplete records of vital signs and progress notes, and, most disturbingly, increased likelihood of preventable adverse events - remains unclear.

With the emergence of multidrug-resistant Gramnegative organisms and novel viruses, the inevitable question being put to IPAC practitioners is whether colonized patients should be identified by screening and isolated to minimize transmission to other patients. Given the lack of high-quality evidence, current practice is highly variable - some institutions implement selective surveillance and isolation of patients in the ICU and nonICU settings, and other institutions isolate only patients identified with infections secondary to these pathogens.

One argument for enhanced precautions follows the precautionary Hippocratic principle of primum non nocere (first, do no harm), which has been central to IPAC since Semmelweiss's time. This fundamental principle, however, has been extrapolated to justify enhanced IPAC measures against drug-resistant organisms (as they are also considered preventable infections) [31,46]. Hand hygiene clearly does not harm patients and is almost certainly beneficial. However, barrier precautions to contain and protect $\mathrm{HCW}$ and other patients from infection with drug-resistant or virulent organisms may arguably cause harm to those patients under isolation.

Until there is evidence that isolating patients with multidrug-resistant organisms is of benefit to the patient and $\mathrm{HCW}$, and that such interventions are cost-effective in reducing the spread of disease (rather than colonization) among critically ill patients, it is difficult to justify enhanced surveillance and precaution strategies to the primary healthcare team without further proof [47]. There is no question that controlling an outbreak is costly to the institution; however, it is not enough to use MRSA control as a prototypic approach for all emerging infections. IPAC measures should take into consideration the setting, epidemiology, virulence factors, mode of transmission and degree of transmissibility of various pathogens: treatment options and strategies for prevention and control at the patient and administrative levels may be completely different among pathogenic organisms and depending on the availability of local resources. 
Given the recent focus on healthcare financing, IPAC measures are being increasingly scrutinized in terms of costs to the hospital. Barrier precautions may require new money but resources may also be found by reprioritizing the hospital's budget, with the focus on preventing hospital-associated infections. Regardless, it is important to critically appraise and promote evidencebased practices that are not only beneficial to patients but are also justified in terms of costs saved to the institution. While some IPAC interventions have been shown to decrease rates of transmission of nosocomial pathogens, there are few studies beyond MRSA and VRE that examine the economic impact of routinely implementing such measures, including droplet isolation for critically ill patients with a cough or airborne precautions where the index of suspicion for tuberculosis is low, or screening for and isolation of asymptomatic patients with extended-spectrum $\beta$-lactamases. Given that IPAC interventions are poorly followed and may not result in reduced transmission rates, further research is important in these areas to determine whether these measures should remain the standard of care. Where the evidence is lacking, especially in the case of novel pathogens, close collaboration, research and innovation among critical care, infection control, infectious diseases, clinical microbiology and nursing staff are essential for the success of preventing and controlling infections for critically ill patients.

\section{Abbreviations}

HCW, healthcare workers; IPAC, infection prevention and control; MRSA, methicillin-resistant Staphylococcus aureus; PCR, polymerase chain reaction; VRE, vancomycin-resistant Enterococcus.

\section{Competing interests}

The authors declare that they have no competing interests.

\section{Author details}

'Division of Infectious Diseases, Department of Pediatrics, The Hospital for Sick Children, 555 University Avenue, Toronto, ON M5G 1X8, Canada. ${ }^{2}$ Division of Infectious Diseases, Department of Medicine, Mount Sinai Hospital and University Health Network; Department of Medicine, University of Toronto; Mount Sinai

Hopsital, 600 University Avenue, Suit 415, Toronto, ON M5G 1X5, Canada.

Published: 19 January 2012

\section{References}

1. Langmuir AD: The Epidemic Intelligence Service of the Center for Disease Control. Public Health Rep 1980, 95:470-477.

2. Gould IM: Alexander Gordon, puerperal sepsis, and modern theories of infection control - Semmelweis in perspective. Lancet Infect Dis 2010, 10:275-278.

3. Holmes O: The contagiousness of puerperal fever. N Engl Q J Med Surg 1843, 1:501-530.

4. Newsom SW: Pioneers in infection control. Ignaz Philipp Semmelweis. J Hosp Infect 1993, 23:175-187.

5. Nightingale F: Notes on Nursing: What It Is, and What It is Not. 1st ed. London: Harrison; 1859.

6. Aboelela SW, Saiman L, Stone P, Lowy FD, Quiros D, Larson E: Effectiveness of barrier precautions and surveillance cultures to control transmission of multidrug-resistant organisms: a systematic review of the literature. Am J Infect Control 2006, 34:484-494.
7. Pittet D, Tarara D, Wenzel RP: Nosocomial bloodstream infection in critically ill patients. Excess length of stay, extra costs, and attributable mortality. JAMA 1994, 271:1598-1601.

8. Burgmann H, Hiesmayr JM, Savey A, Bauer P, Metnitz B, Metnitz PG: Impact of nosocomial infections on clinical outcome and resource consumption in critically ill patients. Intensive Care Med 2010, 36:1597-1601.

9. Hospital Infections Program, National Center for Infectious Diseases, Centers for Disease Control and Prevention, Public Health Service, US Department of Health and Human Services: National Nosocomial Infections Surveillance (NNIS) System report, data summary from January 1990-May 1999, issued June 1999. Am J Infect Control 1999, 27:520-532.

10. Klevens RM, Edwards JR, Tenover FC, McDonald LC, Horan T, Gaynes R: Changes in the epidemiology of methicillin-resistant Staphylococcus aureus in intensive care units in US hospitals, 1992-2003. Clin Infect Dis 2006, 42:389-391.

11. Cosgrove SE, Sakoulas G, Perencevich EN, Schwaber MJ, Karchmer AW, Carmeli Y: Comparison of mortality associated with methicillin-resistant and methicillin-susceptible Staphylococcus aureus bacteremia: a metaanalysis. Clin Infect Dis 2003, 36:53-59.

12. Engemann JJ, Carmeli Y, Cosgrove SE, Fowler VG, Bronstein MZ, Trivette SL, Briggs JP, Sexton DJ, Kaye KS: Adverse clinical and economic outcomes attributable to methicillin resistance among patients with Staphylococcus aureus surgical site infection. Clin Infect Dis 2003, 36:592-598.

13. Jernigan JA, Titus MG, Groschel DH, Getchell-White S, Farr BM: Effectiveness of contact isolation during a hospital outbreak of methicillin-resistant Staphylococcus aureus. Am J Epidemiol 1996, 143:496-504.

14. Muto CA, Jernigan JA, Ostrowsky BE, Richet HM, Jarvis WR, Boyce JM, Farr BM: SHEA guideline for preventing nosocomial transmission of multidrugresistant strains of Staphylococcus aureus and enterococcus. Infect Control Hosp Epidemiol 2003, 24:362-386.

15. Burton DC, Edwards JR, Horan TC, Jernigan JA, Fridkin SK: Methicillinresistant Staphylococcus aureus central line-associated bloodstream infections in US intensive care units, 1997-2007. JAMA 2009, 301:727-736.

16. Doebbeling BN, Stanley GL, Sheetz CT, Pfaller MA, Houston AK, Annis L, Li N, Wenzel RP: Comparative efficacy of alternative hand-washing agents in reducing nosocomial infections in intensive care units. N Engl J Med 1992, 327:88-93.

17. Pittet D, Hugonnet S, Harbarth S, Mourouga P, Sauvan V, Touveneau S, Perneger TV: Effectiveness of a hospital-wide programme to improve compliance with hand hygiene. Infection Control Programme. Lancet 2000, 356:1307-1312.

18. Rosenthal VD, Guzman S, Safdar N: Reduction in nosocomial infection with improved hand hygiene in intensive care units of a tertiary care hospital in Argentina. Am J Infect Control 2005, 33:392-397.

19. Srinivasan A, Song X, Ross T, Merz W, Brower R, Perl TM: A prospective study to determine whether cover gowns in addition to gloves decrease nosocomial transmission of vancomycin-resistant enterococci in an intensive care unit. Infect Control Hosp Epidemiol 2002, 23:424-428.

20. Puzniak LA, Leet T, Mayfield J, Kollef M, Mundy LM: To gown or not to gown: the effect on acquisition of vancomycin-resistant enterococci. Clin Infect Dis 2002, 35:18-25.

21. Pronovost P, Needham D, Berenholtz S, Sinopoli D, Chu H, Cosgrove S, Sexton B, Hyzy R, Welsh R, Roth G, Bander J, Kepros J, Goeschel C: An intervention to decrease catheter-related bloodstream infections in the ICU. N Eng/J Med 2006, 355:2725-2732.

22. Jain R, Kralovic SM, Evans ME, Ambrose M, Simbartl LA, Obrosky DS, Render ML, Freyberg RW, Jernigan JA, Muder RR, Miller LJ, Roselle GA: Veterans Affairs initiative to prevent methicillin-resistant Staphylococcus aureus infections. N Engl J Med 2011, 364:1419-1430.

23. Huang SS, Yokoe DS, Hinrichsen VL, Spurchise LS, Datta R, Miroshnik I, Platt R: Impact of routine intensive care unit surveillance cultures and resultant barrier precautions on hospital-wide methicillin-resistant Staphylococcus aureus bacteremia. Clin Infect Dis 2006, 43:971-978.

24. Thompson RL, Cabezudo I, Wenzel RP: Epidemiology of nosocomial infections caused by methicillin-resistant Staphylococcus aureus. Ann Intern Med 1982, 97:309-317.

25. Pan A, Carnevale G, Catenazzi P, Colombini P, Crema L, Dolcetti L, Ferrari L, Mondello P, Signorini L, Tinelli C, Roldan EQ, Carosi G: Trends in methicillinresistant Staphylococcus aureus (MRSA) bloodstream infections: effect of the MRSA 'search and isolate' strategy in a hospital in Italy with hyperendemic MRSA. Infect Control Hosp Epidemiol 2005, 26:127-133. 
26. Digiovine B, Chenoweth C, Watts C, Higgins M: The attributable mortality and costs of primary nosocomial bloodstream infections in the intensive care unit. Am J Respir Crit Care Med 1999, 160:976-981.

27. Kirkland KB, Weinstein JM: Adverse effects of contact isolation. Lancet 1999, 354:1177-1178.

28. Gagne D, Bedard G, Maziade PJ: Systematic patients' hand disinfection: impact on methicillin-resistant Staphylococcus aureus infection rates in a community hospital. J Hosp Infect 2010, 75:269-272.

29. Karchmer TB, Durbin LJ, Simonton BM, Farr BM: Cost-effectiveness of active surveillance cultures and contact/droplet precautions for control of methicillin-resistant Staphylococcus aureus. J Hosp Infect 2002, 51:126-132.

30. Chaix C, Durand-Zaleski I, Alberti C, Brun-Buisson C: Control of endemic methicillin-resistant Staphylococcus aureus: a cost-benefit analysis in an intensive care unit. JAMA 1999, 282:1745-1751.

31. Muto CA, Giannetta ET, Durbin L, Simonton BM, Farr BM: Cost-effectiveness of perirectal surveillance cultures for controlling vancomycin-resistant Enterococcus. Infect Control Hosp Epidemiol 2002, 23:429-435.

32. Montecalvo MA, Jarvis WR, Uman J, Shay DK, Petrullo C, Horowitz HW, Wormser GP: Costs and savings associated with infection control measures that reduced transmission of vancomycin-resistant enterococci in an endemic setting. Infect Control Hosp Epidemiol 2001, 22:437-442.

33. Saint S, Higgins LA, Nallamothu BK, Chenoweth C: Do physicians examine patients in contact isolation less frequently? A brief report. Am J Infect Control 2003, 31:354-356.

34. Evans HL, Shaffer MM, Hughes MG, Smith RL, Chong TW, Raymond DP, Pelletier SJ, Pruett TL, Sawyer RG: Contact isolation in surgical patients: a barrier to care? Surgery 2003, 134:180-188.

35. Maunder R, Hunter J, Vincent L, Bennett J, Peladeau N, Leszcz M, Sadavoy J, Verhaeghe LM, Steinberg R, Mazzulli T: The immediate psychological and occupational impact of the 2003 SARS outbreak in a teaching hospital. CMAJ 2003, 168:1245-1251

36. Nickell LA, Crighton EJ, Tracy CS, Al-Enazy H, Bolaji Y, Hanjrah S, Hussain A, Makhlouf S, Upshur RE: Psychosocial effects of SARS on hospital staff: survey of a large tertiary care institution. CMAJ 2004, 170:793-798.

37. Stelfox HT, Bates DW, Redelmeier DA: Safety of patients isolated for infection control. JAMA 2003, 290:1899-1905.

38. Koss WG, Khalili TM, Lemus JF, Chelly MM, Margulies DR, Shabot MM: Nosocomial pneumonia is not prevented by protective contact isolation in the surgical intensive care unit. Am Surg 2001, 67:1140-1144

39. Loeb M, Dafoe N, Mahony J, John M, Sarabia A, Glavin V, Webby R, Smieja M, Earn DJ, Chong S, Webb A, Walter SD: Surgical mask vs N95 respirator for preventing influenza among health care workers: a randomized trial. JAMA 2009, 302:1865-1871.

40. Johnson DF, Druce JD, Birch C, Grayson ML: A quantitative assessment of the efficacy of surgical and $\mathrm{N} 95$ masks to filter influenza virus in patients with acute influenza infection. Clin Infect Dis 2009, 49:275-277.

41. Gralton J, McLaws ML: Protecting healthcare workers from pandemic influenza: N95 or surgical masks? Crit Care Med 2010, 38:657-667.

42. Bonten MJ, Slaughter S, Ambergen AW, Hayden MK, van Voorhis J, Nathan C, Weinstein RA: The role of 'colonization pressure' in the spread of vancomycin-resistant enterococci: an important infection control variable. Arch Intern Med 1998, 158:1127-1132.

43. Slaughter S, Hayden MK, Nathan C, Hu TC. Rice T, Van Voorhis J, Matushek M, Franklin C, Weinstein RA: A comparison of the effect of universal use of gloves and gowns with that of glove use alone on acquisition of vancomycin-resistant enterococci in a medical intensive care unit. Ann Intern Med 1996, 125:448-456.

44. Huskins WC, Huckabee CM, O'Grady NP, Murray P,Kopetskie H, Zimmer L, Walker ME, Sinkowitz-Cochran RL, Jernigan JA, Samore M, Wallace D, Goldmann DA: Intervention to reduce transmission of resistant bacteria in intensive care. N Engl J Med 2011, 364:1407-1418.

45. Cohen E, Austin J, Weinstein M, Matlow A, Redelmeier DA: Care of children isolated for infection control: a prospective observational cohort study. Pediatrics 2008, 122:e411-e415.

46. Wenzel RP, Nettleman MD, Jones RN, Pfaller MA: Methicillin-resistant Staphylococcus aureus: implications for the 1990s and effective control measures. Am J Med 1991, 91:221S-227S.

47. Siegel JD, Rhinehart E, Jackson M, Chiarello L, and the Healthcare Infection Control Practices Advisory Committee: Guideline for Isolation Precautions: Preventing Transmission of Infectious Agents in Healthcare Settings. 2007. [http://www.cdc.gov/hicpac/2007|P/2007isolationPrecautions.html] last accessed November 24, 2011.

doi:10.1186/cc10532

Cite this article as: Thampi N, Morris AM: Pro/Con debate: Are barrier precautions cost-effective in improving patient outcomes in the intensive care unit? Critical Care 2012, 16:202. 